\title{
Correlation Between Bowel Preparation and the Adenoma Detection Rate in Screening Colonoscopy
}

\author{
Jung Hun Park, Sang Jin Kim, Jong Hee Hyun, Kyung Su Han, Byung Chang Kim, Chang Won Hong, \\ Sang-Jeon Lee ${ }^{1}$, Dae Kyung Sohn \\ Center for Colorectal Cancer, Research Institute and Hospital, National Cancer Center, Goyang; ${ }^{1}$ Department of Surgery, Chungbuk National \\ University College of Medicine, Chungbuk National University Hospital, Cheongju, Korea
}

Purpose: The adenoma detection rate is commonly used as a measure of the quality of colonoscopy. This study assessed both the association between the adenoma detection rate and the quality of bowel preparation and the risk factors associated with the adenoma detection rate in screening colonoscopy.

Methods: This retrospective analysis involved 1,079 individuals who underwent screening colonoscopy at the National Cancer Center between December 2012 and April 2014. Bowel preparation was classified by using the Aronchick scale. Individuals with inadequate bowel preparations $(\mathrm{n}=47,4.4 \%)$ were excluded because additional bowel preparation was needed. The results of 1,032 colonoscopies were included in the analysis.

Results: The subjects' mean age was 53.1 years, and 657 subjects $(63.7 \%)$ were men. The mean cecal intubation time was 6.7 minutes, and the mean withdrawal time was 8.7 minutes. The adenoma and polyp detection rates were $28.1 \%$ and $41.8 \%$, respectively. The polyp, adenoma, and advanced adenoma detection rates did not correlate with the quality of bowel preparation. The multivariate analysis showed age $\geq 60$ years (hazard ratio [HR], 1.42; 95\% confidence interval [CI], 1.02-1.97; P = 0.040), body mass index $\geq 25 \mathrm{~kg} / \mathrm{m}^{2}$ (HR, 1.56; 95\% CI, 1.17-2.08; P = 0.002) and current smoking (HR, 1.44; 95\% CI, 1.01-2.06; P = 0.014) to be independent risk factors for adenoma detection.

Conclusion: The adenoma detection rate was unrelated to the quality of bowel preparation for screening colonoscopy. Older age, obesity, and smoking were independent risk factors for adenoma detection.

\section{Keywords: Adenoma detection rate; Bowel preparation; Colonoscopy}

\section{INTRODUCTION}

In 2012, 28,988 individuals in Korea were newly diagnosed with colorectal cancer. Colorectal cancer has become the second most common cancer in Korean men and the third most common in Korean women, and its incidence has continued to increase in both sexes [1]. Colonoscopy has become the gold standard

Received: August 24, 2016 - Accepted: March 10, 2017

Correspondence to: Dae Kyung Sohn, M.D.

Center for Colorectal Cancer, Research Institute and Hospital, National

Cancer Center, 323 Ilsan-ro, Ilsandong-gu, Goyang 10408, Korea

Tel: +82-31-920-1636, Fax: +82-31-920-1148

E-mail: gsgsbal@ncc.re.kr

(c) 2017 The Korean Society of Coloproctology

This is an open-access article distributed under the terms of the Creative Commons Attribution NonCommercial License (http://creativecommons.org/licenses/by-nc/4.0) which permits unrestricted noncommercial use, distribution, and reproduction in any medium, provided the original work is properly cited. screening test for colorectal cancer. Because a colonic adenoma is related to interval cancer [2], colonoscopic removal of adenomatous polyps reduces the incidence of and the mortality from colorectal cancer [3-5]. The U.S. Multi-Society Task Force on Colorectal Cancer has targeted the adenoma detection rate for quality improvement during colonoscopy [6], with the adenoma detection rate being regarded as an important indicator of the quality of colonoscopy [7-9].

The diagnostic accuracy and the therapeutic safety of screening colonoscopy depend on the quality of bowel preparation. Adequate bowel preparation is essential for a successful inspection of the colonic mucosa [10-12]. Little is known about the impact of the quality of bowel preparation on the adenoma detection rate during screening colonoscopy. This study was, therefore, designed both to evaluate the association between the adenoma detection rate and the quality of bowel preparation and to assess the risk factors associated with adenoma detection. 


\section{METHODS}

\section{Study population}

The medical charts of 1,079 individuals who had undergone screening colonoscopies at the National Cancer Center in Korea between December 2012 and April 2014 were retrospectively reviewed. Subjects with inadequate bowel preparations $(n=47$, $4.4 \%$ ) were excluded because additional bowel preparation was needed. Thus, colonoscopies of 1,032 individuals were included in the analysis. Each started bowel preparation with PEG-3350 plus ascorbic acid (Coolprep, Taejoon, Seoul, Korea) between 6:00 $\mathrm{PM}$ and 10:00 PM on the evening before the colonoscopy. Colonoscopies were performed between 9:00 AM and 12:00 noon. This study was approved by the Institutional Review Board of National Cancer Center (NCC2014-0132).

\section{Definitions}

Bowel preparations were classified by using the Aronchick scale (Fig. 1) based on the proportion of fluid and remnant stool [13]. Excellent was defined as the presence of a small volume of clear liquid or visualization of greater than $95 \%$ of the surface, good as a large volume of clear liquid covering $5 \%$ to $25 \%$ of the surface but visualization of greater than $90 \%$ of the surface, fair as the presence of some semisolid stool that could be suctioned or washed away but visualization of greater than $90 \%$ of the surface, poor as the presence of semisolid stool that could not be suctioned or washed away and visualization of less than $90 \%$ of the surface, and inadequate as solid stool that impeded visualization, requiring repeat preparation and colonoscopy.

The adenoma and the polyp detection rates were defined as the proportion of subjects with at least one adenoma or polyp divided by the total number of colonoscopies. Advanced adenomas are typically defined as adenomas greater than or equal to $1 \mathrm{~cm}$ in diameter or those with villous components (tubulovillous or villous) or with high-grade or severe dysplasia [14].

\section{Statistical analysis}

Continuous variables were compared using the Student t-test whereas categorical variables were compared using the chi-square or Fisher exact test. Logistic regression was used for multivariate analyses to determine the independent risk factors for adenoma detection. An analysis of variance was used to compare the quality of colonoscopy between bowel preparation groups. A P-value of less than 0.05 was considered statistically significant. All statistical analyses were performed using the SPSS ver. 14.0 ( SPSS Inc., Chicago, IL, USA).

\section{RESULTS}

\section{Baseline characteristics}

The baseline characteristics of the 1,032 individuals included in this study are shown in Table 1 . The mean age of these subjects was 53.1 years; $657(63.7 \%)$ were men and $375(36.3 \%)$ were women. The mean body mass index was $24.0 \mathrm{~kg} / \mathrm{m}^{2}$. The mean cecal intubation time was 6.7 minutes, and the mean withdrawal time was 8.7 minutes. Bowel preparation was excellent, good, fair, and poor in $17(1.6 \%), 640$ (62.0\%), 371 (35.9\%), and 4 subjects $(0.4 \%)$, respectively.

\section{Bowel preparation and quality of colonoscopy}

The overall rates of adenoma, polyp, and advanced adenoma detection were $28.1 \%, 41.8 \%$, and $3.7 \%$, respectively. The adenoma detection rate was $31.8 \%$ in men and $21.6 \%$ in women. The polyp detection rates in subjects who underwent excellent, good, fair, and poor bowel preparation were $35.3 \%, 42.8 \%, 40.4 \%$, and $25.0 \%$, respectively $(\mathrm{P}=0.726)$. The adenoma detection rates in these groups were $23.5 \%, 29.1 \%, 27.0 \%$, and $0 \%$, respectively $(\mathrm{P}=$ 0.519 ), and the advanced adenoma detection rates were $5.9 \%$, $4.1 \%, 3.0 \%$, and $0 \%$, respectively $(\mathrm{P}=0.758)$. The cecal intubation time was significantly affected by bowel preparation $(\mathrm{P}=0.004)$, being longer in subjects with poor bowel preparation. In contrast,

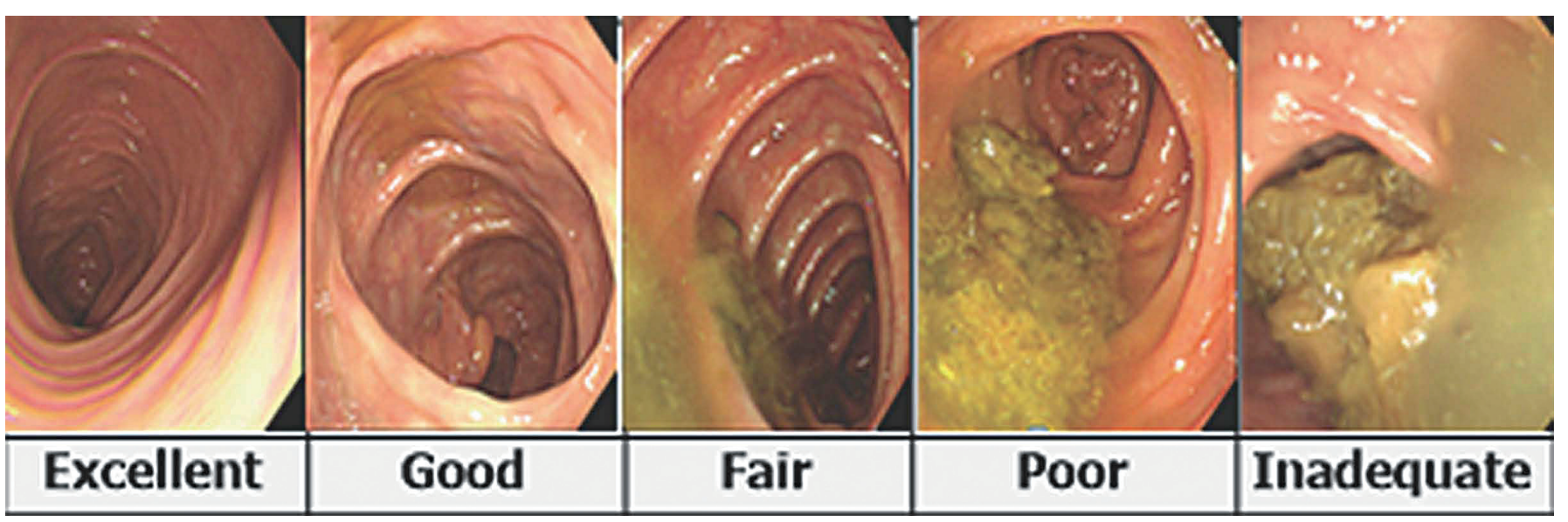

Fig. 1. Aronchick bowel preparation scale. 
withdrawal time was not significantly associated with the quality of bowel preparation $(\mathrm{P}=0.063)$ (Table 2$)$.

\section{Risk factors for the adenoma detection rate}

Table 3 shows the results of the univariate and the multivariate analyses of the risk factors for adenoma detection. The univariate analysis showed that male sex, age $\geq 60$ years, body mass index $(\mathrm{BMI}) \geq 25 \mathrm{~kg} / \mathrm{m}^{2}$, and current smoking were significant risk factors for adenoma detection. The multivariate analysis showed that age $\geq 60$ years (hazard ratio [HR], 1.42; 95\% confidence interval [CI], 1.02-1.97; P = 0.040), BMI $\geq 25 \mathrm{~kg} / \mathrm{m}^{2}$ ( $\mathrm{HR}, 1.56 ; 95 \% \mathrm{CI}$, 1.17-2.08; $\mathrm{P}=0.002)$ and current smoking (HR, 1.44; 95\% CI,

Table 1. Baseline characteristics of the study subjects $(n=1,032)$

\begin{tabular}{lc}
\hline Characteristic & Value \\
\hline Age $(\mathrm{yr})$ & $53.1 \pm 8.7$ \\
Sex, men : women & $657: 375$ \\
Body mass index $\left(\mathrm{kg} / \mathrm{m}^{2}\right)$ & $24.0 \pm 2.9$ \\
Smoking & \\
$\quad$ Never & $439(42.5)$ \\
Ex-smoker & $326(31.6)$ \\
Current smoker & $267(25.9)$ \\
Alcohol & \\
No & $274(26.6)$ \\
Yes & $758(73.4)$ \\
Bowel preparation quality & \\
Excellent & $17(1.6)$ \\
Good & $640(62.0)$ \\
Fair & $371(35.9)$ \\
Poor & $4(0.4)$ \\
Cecal intubation time (min) & $6.7 \pm 4.5$ \\
Withdrawal time (min) & $8.7 \pm 3.3$ \\
\hline
\end{tabular}

Values are presented as mean \pm standard deviation, number, or number (\%).
1.01-2.06; $\mathrm{P}=0.014)$ were independent risk factors for adenoma detection.

\section{DISCUSSION}

Previous studies assessing the relationship between the adenoma detection rate and bowel preparation have yielded various outcomes. For example, one study reported that the adenoma detection rate for good bowel preparation was significantly higher than it was for excellent bowel preparation [15] whereas another study found no significant difference between good and excellent bowel preparation [16]. Other studies found that the adenoma detection rate was not significantly different in subjects with fair bowel preparation compared to those with excellent and good bowel preparation $[17,18]$, although the rate of missed adenomas after poor bowel preparation was significantly higher than it was after excellent, good or fair bowel preparation [19]. In contrast, adenoma detection rates were reported to be similar after excellent (24.2\%), good $(26.8 \%)$, fair $(32.1 \%)$, and poor $(22.1 \%)$ bowel preparation [20]. Similarly, this study found that adenoma detection rates were unrelated to the degree of bowel preparation. As the rate of poor bowel preparation $(n=4,0.4 \%)$ was very low, this subgroup was excluded from comparative analyses. Nevertheless, a comparison of three groups of subjects, those with excellent, good, and fair bowel preparation, showed no significant differences in the polyp $(P=0.654)$, the adenoma $(P=0.704)$, and the advanced adenoma $(\mathrm{P}=0.599)$ detection rates.

Bowel preparation was found to be inadequate in 47 subjects, with adenomas detected in 12 of these subjects (25.5\%). Because all subjects with inadequate bowel preparation required repeat bowel preparations, actual bowel preparation grades were changed following the repeat colonoscopies. Overall, these findings suggest that the incidences of colorectal adenomas were similar in subjects with adequate preparation and those with inadequate bowel preparation.

This study also found that age $\geq 60$ years, BMI $\geq 25 \mathrm{~kg} / \mathrm{m}^{2}$, and smoking were independent risk factors for adenoma detection.

Table 2. Bowel preparation and quality of colonoscopy

\begin{tabular}{|c|c|c|c|c|c|}
\hline \multirow{2}{*}{ Variable } & \multicolumn{4}{|c|}{ Quality of bowel preparation ${ }^{\mathrm{a}}$} & \multirow{2}{*}{ P-value } \\
\hline & Excellent $(n=17)$ & Good $(n=640)$ & Fair $(n=371)$ & Poor $(n=4)$ & \\
\hline PDR & $6(35.3)$ & $274(42.8)$ & $150(40.4)$ & $1(25.0)$ & 0.726 \\
\hline ADR & $4(23.5)$ & $186(29.1)$ & $100(27.0)$ & $0(0)$ & 0.519 \\
\hline Advanced ADR ${ }^{b}$ & $1(5.9)$ & $26(4.1)$ & $11(3.0)$ & $0(0)$ & 0.758 \\
\hline \multirow[t]{2}{*}{ Cecal intubation time (min) } & $6.5 \pm 5.4$ & $6.7 \pm 4.5$ & $6.8 \pm 4.3$ & $14.8 \pm 3.6$ & 0.004 \\
\hline & Excellent $(n=11)$ & Good $(n=366)$ & Fair $(n=221)$ & Poor $(n=3)$ & \\
\hline Withdrawal time $(\mathrm{min})^{c}$ & $6.9 \pm 1.7$ & $8.7 \pm 5.7$ & $9.0 \pm 3.6$ & $15.3 \pm 4.2$ & 0.063 \\
\hline
\end{tabular}

Values are presented as number (\%) or mean \pm standard deviation.

PDR, polyp detection rate; $A D R$, adenoma detection rate.

${ }^{a}$ Classified by using the Aronchick scale. ${ }^{b}$ Adenoma $\geq 1 \mathrm{~cm}$ in diameter, with villous components or with high-grade dysplasia. ${ }^{\circ}$ Only in subjects without colorectal polyps. 
Table 3. Multivariate analysis of risk factors associated with the adenoma detection rate

\begin{tabular}{|c|c|c|c|c|}
\hline \multirow{2}{*}{ Variable } & \multirow{2}{*}{ ADR, n (\%) } & \multirow{2}{*}{$\begin{array}{c}\text { Univariate } \\
\text { P-value }\end{array}$} & \multicolumn{2}{|c|}{ Multivariate } \\
\hline & & & HR $(95 \% \mathrm{Cl})$ & P-value \\
\hline Sex & & $<0.001$ & & \\
\hline Female & 81/375 (21.6) & & & \\
\hline Male & 209/657 (31.8) & & & \\
\hline Age (yr) & & 0.025 & & 0.040 \\
\hline$<60$ & 214/809 (26.5) & & 1.00 & \\
\hline$\geq 60$ & $76 / 223(34.1)$ & & $1.42(1.02-1.97)$ & \\
\hline Body mass index $\left(\mathrm{kg} / \mathrm{m}^{2}\right)$ & & $<0.001$ & & 0.002 \\
\hline$<25$ & $162 / 672(24.1)$ & & 1.00 & \\
\hline$\geq 25$ & 128/360 (35.6) & & $1.56(1.17-2.08)$ & \\
\hline Smoking & & 0.001 & & 0.014 \\
\hline Never & 99/439 (22.6) & & 1.00 & \\
\hline Ex-smoker & $112 / 326(34.4)$ & & $1.60(1.15-2.23)$ & \\
\hline Current smoker & 79/267 (29.6) & & $1.44(1.01-2.06)$ & \\
\hline Alcohol & & 0.638 & & \\
\hline No & $74 / 274(27.0)$ & & & \\
\hline Yes & $216 / 758(28.5)$ & & & \\
\hline Bowel preparation & & 0.674 & & \\
\hline Excellent & 4/17 (23.5) & & & \\
\hline Good & $186 / 640(29.1)$ & & & \\
\hline Fair & $100 / 371(27.0)$ & & & \\
\hline Poor & $0 / 4(0)$ & & & \\
\hline
\end{tabular}

$\mathrm{ADR}$, adenoma detection rate; $\mathrm{HR}$, hazard ratio; $\mathrm{Cl}$, confidence interval.

Similarly, age over 50 years was found to be associated with a high adenoma detection rate [21], and a multivariate meta-analysis confirmed a positive association between higher BMI and the prevalence of colorectal adenomas [22]. Other studies found that cigarette smoking [23] and male sex [24] were independently associated with a higher adenoma detection rate. Although our study found that the adenoma detection rate was higher in men than in women, the multivariate analysis demonstrated that sex was not an independent risk factor for adenoma detection $(\mathrm{P}=0.240)$.

Characteristics associated with the adenoma detection rate during screening colonoscopy can be classified as patient factors, colonoscopist factors, and endoscope factors [25]. Patient-associated factors include male sex, age, $\mathrm{BMI} \geq 25 \mathrm{~kg} / \mathrm{m}^{2}$, familial and personal history of colorectal polyps and colorectal cancer, positive fecal occult blood test results, cigarette smoking, alcohol consumption, and bowel preparation [21-25]. Colonoscopist-associated factors include the cecal intubation time, the withdrawal time, the colonoscopist's training and experience, the use of intravenous antispasmodics, and the procedure start time [26-28]. Endoscope factors include the generation of the instrument used [25]. The risk factors for colorectal adenoma detection, including older age, being overweight, male sex, and smoking, are also risk factors for colorectal adenomas [23, 29].

The rates of detection of any neoplasia and of advanced neoplasia have been reported to be higher in subjects with mean withdrawal times longer than or equal to 6 minutes than in those with withdrawn times shorter than 6 minutes [27]. The mean withdrawal time of our study subjects was 8.7 minutes, with both the cecal intubation time and the withdrawal time being longer in subjects with poor bowel preparation. These longer times are likely due to poor visualization during colonoscope insertion, suggesting that suction and irrigation may improve visualization of the colonic mucosa during instrument withdrawal. The cecal intubation time $(\mathrm{P}=0.863)$ and the withdrawal time $(\mathrm{P}=0.359)$, however, were similar in subjects with excellent, good, and fair bowel preparation.

The adenoma detection rates during screening colonoscopy have been reported to range from $9.4 \%$ to $37.5 \%$ [30]. The overall adenoma detection rate among subjects in this study was $28.1 \%$. The American Society of Gastrointestinal Endoscopy and the American College of Gastroenterology Task Force recommended minimal adenoma detection rates of more than $25 \%$ for average- 
risk men aged 50 years or older and of more than $15 \%$ for average-risk women aged 50 years or older $[6,8]$. The adenoma detection rates of men and women in this study were even higher, being $31.8 \%$ and $21.6 \%$, respectively.

This study had several limitations. First, it was retrospective in design and involved individuals at a single institution. Second, the sample sizes of the four bowel preparation groups were uneven, with few subjects in the poor bowel preparation group, suggesting that this group may have had an outsized effect on our study results. Third, because this study did not include all possible risk factors associated with adenoma detection, the results of this study may not be generalizable.

In conclusion, the adenoma detection rate was unrelated to the quality of bowel preparation for screening colonoscopy. Independent risk factors for the adenoma detection rate included older age, obesity, and smoking.

\section{CONFLICT OF INTEREST}

No potential conflicts of interest relevant to this article were reported.

\section{ACKNOWLEDGMENTS}

This work was supported by the National Cancer Center Grant (NCC-1510150).

\section{REFERENCES}

1. Jung KW, Won YJ, Kong HJ, Oh CM, Cho H, Lee DH, et al. Cancer statistics in Korea: incidence, mortality, survival, and prevalence in 2012. Cancer Res Treat 2015;47:127-41.

2. Kaminski MF, Regula J, Kraszewska E, Polkowski M, Wojciechowska U, Didkowska J, et al. Quality indicators for colonoscopy and the risk of interval cancer. N Engl J Med 2010;362: 1795-803.

3. Zauber AG, Winawer SJ, O'Brien MJ, Lansdorp-Vogelaar I, van Ballegooijen M, Hankey BF, et al. Colonoscopic polypectomy and long-term prevention of colorectal-cancer deaths. N Engl J Med 2012;366:687-96.

4. Winawer SJ, Zauber AG, Ho MN, O’Brien MJ, Gottlieb LS, Sternberg SS, et al. Prevention of colorectal cancer by colonoscopic polypectomy. The National Polyp Study Workgroup. N Engl J Med 1993;329:1977-81.

5. Jacob BJ, Moineddin R, Sutradhar R, Baxter NN, Urbach DR. Effect of colonoscopy on colorectal cancer incidence and mortality: an instrumental variable analysis. Gastrointest Endosc 2012;76: 355-64.e1.

6. Rex DK, Bond JH, Winawer S, Levin TR, Burt RW, Johnson DA, et al. Quality in the technical performance of colonoscopy and the continuous quality improvement process for colonoscopy: recommendations of the U.S. Multi-Society Task Force on Colorectal Cancer. Am J Gastroenterol 2002;97:1296-308.
7. Millan MS, Gross P, Manilich E, Church JM. Adenoma detection rate: the real indicator of quality in colonoscopy. Dis Colon Rectum 2008;51:1217-20.

8. Rex DK, Petrini JL, Baron TH, Chak A, Cohen J, Deal SE, et al. Quality indicators for colonoscopy. Am J Gastroenterol 2006;101 :873-85.

9. Bretagne JF, Ponchon T. Do we need to embrace adenoma detection rate as the main quality control parameter during colonoscopy? Endoscopy 2008;40:523-8.

10. Wexner SD, Beck DE, Baron TH, Fanelli RD, Hyman N, Shen B, et al. A consensus document on bowel preparation before colonoscopy: prepared by a task force from the American Society of Colon and Rectal Surgeons (ASCRS), the American Society for Gastrointestinal Endoscopy (ASGE), and the Society of American Gastrointestinal and Endoscopic Surgeons (SAGES). Gastrointest Endosc 2006;63:894-909.

11. Harewood GC, Sharma VK, de Garmo P. Impact of colonoscopy preparation quality on detection of suspected colonic neoplasia. Gastrointest Endosc 2003;58:76-9.

12. Froehlich F, Wietlisbach V, Gonvers JJ, Burnand B, Vader JP. Impact of colonic cleansing on quality and diagnostic yield of colonoscopy: the European Panel of Appropriateness of Gastrointestinal Endoscopy European multicenter study. Gastrointest Endosc 2005;61:378-84.

13. Lorenzo-Zúñiga V, Moreno-de-Vega V, Boix J. Preparation for colonoscopy: types of scales and cleaning products. Rev Esp Enferm Dig 2012;104:426-31.

14. Lieberman DA, Rex DK, Winawer SJ, Giardiello FM, Johnson DA, Levin TR, et al. Guidelines for colonoscopy surveillance after screening and polypectomy: a consensus update by the US MultiSociety Task Force on Colorectal Cancer. Gastroenterology 2012; 143:844-57.

15. Calderwood AH, Thompson KD, Schroy PC 3rd, Lieberman DA, Jacobson BC. Good is better than excellent: bowel preparation quality and adenoma detection rates. Gastrointest Endosc 2015; 81:691-9.e1.

16. Tholey DM, Shelton CE, Francis G, Anantharaman A, Frankel $\mathrm{RA}$, Shah $\mathrm{P}$, et al. Adenoma detection in excellent versus good bowel preparation for colonoscopy. J Clin Gastroenterol 2015;49: 313-9.

17. Clark BT, Rustagi T, Laine L. What level of bowel prep quality requires early repeat colonoscopy: systematic review and metaanalysis of the impact of preparation quality on adenoma detection rate. Am J Gastroenterol 2014;109:1714-23.

18. Sherer EA, Imler TD, Imperiale TF. The effect of colonoscopy preparation quality on adenoma detection rates. Gastrointest Endosc 2012;75:545-53.

19. Kim JS, Kang SH, Moon HS, Lee ES, Kim SH, Sung JK, et al. Impact of bowel preparation quality on adenoma identification during colonoscopy and optimal timing of surveillance. Dig Dis Sci 2015;60:3092-9.

20. Rai T, Navaneethan U, Gohel T, Podugu A, Thota PN, Kiran RP, 
et al. Effect of quality of bowel preparation on quality indicators of adenoma detection rates and colonoscopy completion rates. Gastroenterol Rep (Oxf) 2016;4:148-53.

21. Barret M, Boustiere C, Canard JM, Arpurt JP, Bernardini D, Bulois $\mathrm{P}$, Factors associated with adenoma detection rate and diagnosis of polyps and colorectal cancer during colonoscopy in France: results of a prospective, nationwide survey. PLoS One 2013;8:e68947.

22. Okabayashi K, Ashrafian H, Hasegawa H, Yoo JH, Patel VM, Harling $\mathrm{L}$, et al. Body mass index category as a risk factor for colorectal adenomas: a systematic review and meta-analysis. Am J Gastroenterol 2012;107:1175-85.

23. Martínez ME, McPherson RS, Annegers JF, Levin B. Cigarette smoking and alcohol consumption as risk factors for colorectal adenomatous polyps. J Natl Cancer Inst 1995;87:274-9.

24. Schramm C, Mbaya N, Franklin J, Demir M, Kuetting F, Toex U, et al. Patient- and procedure-related factors affecting proximal and distal detection rates for polyps and adenomas: results from 1603 screening colonoscopies. Int J Colorectal Dis 2015;30:171522.

25. Adler A, Wegscheider K, Lieberman D, Aminalai A, Aschenbeck J,
Drossel R, et al. Factors determining the quality of screening colonoscopy: a prospective study on adenoma detection rates, from 12,134 examinations (Berlin colonoscopy project 3, BECOP-3). Gut 2013;62:236-41.

26. Coe SG, Crook JE, Diehl NN, Wallace MB. An endoscopic quality improvement program improves detection of colorectal adenomas. Am J Gastroenterol 2013;108:219-26.

27. Barclay RL, Vicari JJ, Doughty AS, Johanson JF, Greenlaw RL. Colonoscopic withdrawal times and adenoma detection during screening colonoscopy. N Engl J Med 2006;355:2533-41.

28. Lee TJ, Rees CJ, Blanks RG, Moss SM, Nickerson C, Wright KC, et al. Colonoscopic factors associated with adenoma detection in a national colorectal cancer screening program. Endoscopy 2014; 46:203-11.

29. Hassan C, Pickhardt PJ, Marmo R, Choi JR. Impact of lifestyle factors on colorectal polyp detection in the screening setting. Dis Colon Rectum 2010;53:1328-33.

30. Adler A, Aschenbeck J, Yenerim T, Mayr M, Aminalai A, Drossel $\mathrm{R}$, et al. Narrow-band versus white-light high definition television endoscopic imaging for screening colonoscopy: a prospective randomized trial. Gastroenterology 2009;136:410-6.e1. 